\title{
Entrevista motivacional no autogerenciamento de cuidados a adolescentes com diabetes tipo 1: revisão integrativa
}

\author{
Motivational interviewing and self-management among adolescents with type 1 diabetes: an integrative \\ review
}

\author{
Entrevista motivacional en la autogestión de los cuidados a adolescentes con diabetes tipo 1: revisión \\ integradora
}

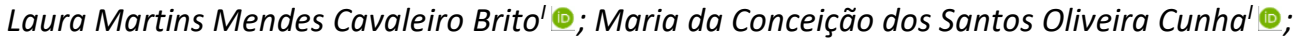 \\ Amanda Newle Sousa Silva’@; Maria Veraci Oliveira Queiroz’ @
}

'Universidade Estadual do Ceará, Fortaleza, CE, Brasil

\begin{abstract}
RESUMO
Objetivo: analisar evidências científicas sobre entrevista motivacional e sua influência no autogerenciamento do cuidado do adolescente com diabetes mellitus tipo 1. Método: revisão integrativa cuja busca aconteceu em junho de 2020 nas bases de dados LILACS, PubMed, CINAHL, Scopus e Web of Science para atender a pergunta: Quais evidências científicas sobre entrevista motivacional e sua influência no autogerenciamento do cuidado ao adolescente com diabetes mellitus tipo 1 ? Todos os artigos identificados estavam no idioma inglês e publicados entre 2003 e 2020. Resultados: entre os dez estudos incluídos, nove foram realizados nos Estados Unidos e um no Reino Unido. As pesquisas tiveram métodos diversificados na aplicação da entrevista motivacional em circunstâncias diferentes e os resultados mostraram impactos positivos no autogerenciamento dos cuidados de adolescentes com DM1, principalmente em relação ao controle glicêmico e/ou nos níveis de hemoglobina glicada. Conclusão: a entrevista motivacional favorece o autogerenciamento do cuidado e autonomia do adolescente com diabetes. Descritores: Diabetes Mellitus Tipo 1; Adolescente; Entrevista Motivacional; Autoeficácia; Autogestão.
\end{abstract}

\begin{abstract}
Objective: this study examined scientific evidence on motivational interviewing and its influence on self-management among adolescents with type-1 diabetes mellitus (DM1). Methods: the survey for this integrative review took place in June 2020 in the LILACS, PubMed, CINAHL, Scopus, and Web of Science databases to answer the question: What scientific evidence is available on motivational interviewing and its influence on self-management among adolescents with DM1? All the articles found were in English and published between 2003 and 2020. Results: of the ten studies included, nine were conducted in the United States and one in the United Kingdom. The studies used different methods for applying motivational interviewing in differing circumstances, and their findings revealed positive impacts on self-management among adolescents with type-1 diabetes, especially on blood glucose monitoring and/or on glycated hemoglobin levels. Conclusion: motivational interviewing is an intervention that favors self-management and autonomy among adolescents with diabetes.

Descriptors: Diabetes Mellitus, Type1; Adolescent; Motivational Interviewing; Self Efficacy; Self-Management.
\end{abstract}

\section{RESUMEN}

Objetivo: analizar evidencias científicas sobre entrevista motivacional y su influencia en la autogestión del cuidado de adolescentes con diabetes mellitus tipo 1 (DM1). Métodos: revisión integradora llevada a cabo en junio de 2020 en las bases de datos LILACS, PubMed, CINAHL, Scopus y WoS para responder a la pregunta: ¿Cuáles son las evidencias científicas sobre entrevista motivacional y su influencia en la autogestión del cuidado de adolescentes con diabetes mellitus tipo 1? Los artículos encontrados estaban en inglés y fueron publicados entre 2003 y 2020. Resultados: de los diez estudios incluidos, nueve se realizaron en Estados Unidos y uno en Reino Unido. Las investigaciones utilizaron diferentes métodos de aplicación de la entrevista motivacional en diferentes circunstancias y los resultados señalaron impactos positivos en la autogestión de los cuidados de adolescentes con DM1, especialmente cuanto al control glucémico y/o niveles de hemoglobina glucosilada. Conclusión: la entrevista motivacional favorece la autogestión del cuidado y la autonomía de adolescentes con diabetes.

Descriptores: Diabetes Mellitus Tipo 1; Adolescente; Entrevista Motivacional; Autoeficacia; Automanejo.

\section{INTRODUÇÃO}

A prevalência global de Diabetes Mellitus tipo 1 (DM1) em crianças, adolescentes e jovens é de 1.110 .100 casos, sendo a incidência global anual estimada em cerca de 128.900 nessa faixa etária abaixo de 20 anos. Os países com maiores números de casos prevalentes em crianças e adolescentes com idade inferior a 15 são Índia (95,6 mil), Estados Unidos (94,2 mil) e, em terceiro lugar, o Brasil (51,5 mil) ${ }^{1}$.

Em decorrência das mudanças inerentes à fase de desenvolvimento, o adolescente vivencia a formação de sua identidade e suas relações com os pares tornam-se mais importantes do que os relacionamentos com os familiares e 
profissionais, às vezes, permeadas de conflitos $^{2}$. Sugere-se, portanto, que as intervenções de saúde incluam um envolvimento ativo do paciente, a fim de melhorar o autocuidado ${ }^{3}$.

Nesta perspectiva, os profissionais que cuidam do adolescente com DM1 devem utilizar estratégias que visem melhorar o autogerenciamento dos adolescentes no manejo da doença. O autogerenciamento do diabetes requer que os pacientes sejam capazes de conciliar seus recursos e preferências com o tratamento para tornar seu engajamento no cuidado mais positivo ${ }^{4}$.

Destarte, observa-se um crescente interesse em estudar a qualidade de vida, o comportamento emocional e o autocuidado entre pacientes com DM1 ${ }^{3}$. Ressalta-se que uma comunicação motivadora que auxilie uma relação de confiança entre o adolescente, sua família e o profissional de saúde é fundamental para alcançar o manejo adequado da doença. Desta forma, a Entrevista Motivacional (EM) é uma modalidade de intervenção, um estilo de aconselhamento centrado no paciente, no intuito de provocar mudanças de comportamento, ajudando as pessoas identificarem suas próprias atitudes relacionadas ao estado de saúde. A EM segue os princípios fundamentais de expressão da empatia, incorporação à mudança, e, consequentemente, evocação da motivação intrínseca 5 .

O enfermeiro em suas ações de consulta, aconselhamentos e atividades educativas junto ao adolescente com DM1 deve utilizar estratégias que propiciem o autogerenciamento do cuidado resultando em melhoria ao seu bem-estar e a promoção da saúde, característica fundamental do seu trabalho ${ }^{6}$. Para tanto, são necessárias habilidades específicas na condução de estratégias para o alcance de atitudes saudáveis a partir de seus próprios motivos. Diante disso, a EM é uma estratégia útil para trabalhar com a ambivalência sobre a mudança ${ }^{7}$.

Nesse contexto, apresenta-se a síntese do conhecimento disponível sobre a EM e sua influência no autogerenciamento do cuidado de adolescentes com diabetes tipo 1. Vislumbra-se contribuir com decisões na prática clínica, essencialmente, no aconselhamento de adolescentes com DM1, utilizando a entrevista motivacional. Assim, o objetivo desta revisão foi analisar as evidências científicas sobre a entrevista motivacional e sua influência no autogerenciamento do cuidado do adolescente com diabetes mellitus tipo 1.

\section{MÉTODO}

Revisão integrativa da literatura delineada em seis etapas: identificar o tema e selecionar a hipótese ou questão de pesquisa; estabelecer critérios para inclusão e exclusão de estudos/amostragem ou busca na literatura; definir informações a serem extraídas dos estudos selecionados e categorizá-los; avaliar os estudos incluídos para a revisão; interpretar os resultados; e apresentar a síntese dos conhecimentos ${ }^{8,9}$.

A busca na literatura e a seleção dos estudos foram realizadas por duas pesquisadoras de forma independente em julho de 2020, utilizando-se as bases de dados: Scopus (Sci Verse Scopus), PubMed (PubMed Central: PMC), LILACS (Literatura Latino-Americana e do Caribe em Ciências da Saúde), CINAHL (Current Nursing and Allied Health Literature) e Web of Science (Coleção Principal).

Na elaboração da questão da pesquisa utilizou-se a estratégia PICO sendo acrônimo para Patient, Intervention, Comparison, Outcomes ${ }^{10}$. Definiu-se como P (Adolescentes e Diabetes Mellitus); I (entrevista motivacional); C (Não contempla) e O (Autogerenciamento). Assim, a questão norteadora foi: Quais as evidências científicas sobre a entrevista motivacional e sua influência no autogerenciamento do cuidado ao adolescente com diabetes mellitus tipo 1 ?

Os estudos foram identificados com equações de buscas utilizando-se os Descritores em Ciências da Saúde (DeCS/MeSH) com auxílio dos operadores booleanos (AND e OR), conforme apresentado na Figura 1. Optou-se pelo descritor diabetes mellitus para possibilitar ampliação do número de artigos.

\begin{tabular}{|ll|}
\hline Grupos & Equações de buscas \\
\hline População & adolescent OR teen* OR youth* OR juvenile OR "young people" OR young \\
População & diabetes OR "diabetes mellitus" \\
Intervenção & "motivational interviewing" OR "health communication" \\
Resultados & "self efficacy" OR "self-efficacy" OR "self care" OR "self-care" OR "self-management" OR "self \\
& management" \\
\hline
\end{tabular}

FIGURA 1: Estratégia PICO - “Quais as evidências científicas disponíveis sobre a entrevista motivacional e o autogerenciamento do cuidado de adolescente com diabetes mellitus tipo 1?" Fortaleza, CE, Brasil, 2020

Fonte: Elaborado pelas autoras 
Os critérios de inclusão foram: artigos escritos em português, espanhol e inglês publicados e disponíveis eletronicamente ou com acesso permitido no Portal de Periódicos da Coordenação de Aperfeiçoamento de Pessoal de Nível Superior (CAPES), via Comunidade Acadêmica Federada (CAFe). Não foi estabelecido recorte temporal para inclusão dos artigos. Foram excluídos os trabalhos duplicados, editoriais, artigos de opinião e os artigos que não responderam à pergunta norteadora.

Inicialmente, a busca resultou em 111 publicações, potencialmente elegíveis. Em seguida, foram retirados os estudos duplicados, e, após a leitura do título e resumo de cada publicação, restaram 43. Com a aplicação dos critérios de seleção, foram excluídas 33 publicações, obtendo-se a amostra de 10 estudos primários.

A busca e triagem de artigos foram orientadas pela estrutura PRISMA ${ }^{11}$, resumida na Figura 2.

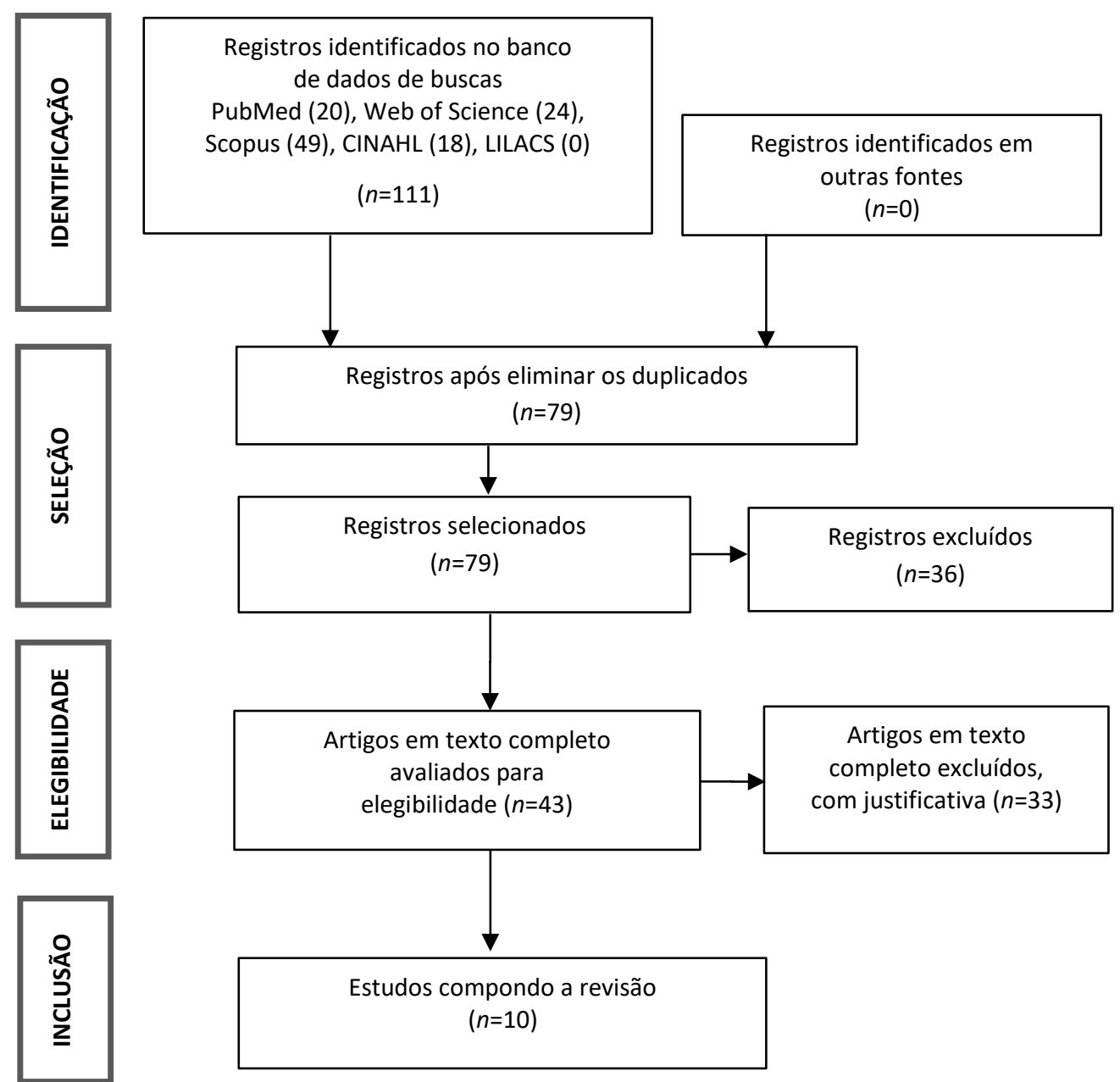

FIGURA 2: Fluxograma do processo de seleção dos estudos primários adaptado do Preferred Reporting Items for Systematic Review and Meta-Analyses (PRISMA). Fortaleza, CE, Brasil, 2020.

Elaborou-se um quadro com os estudos incluídos na revisão com os seguintes dados: título, autor, ano de publicação, país de origem, nível de evidência, objetivo e principais resultados, apresentados na sessão resultados, em ordem decrescente do ano de publicação. Os estudos foram identificados com a letra "E" (E1- artigo 1, E2 - artigo 2 e assim sucessivamente).

Para análise dos estudos levantados, foi adotada a classificação hierárquica das evidências, a fim de classificar a força das evidências diante do objeto estudado. Neste âmbito, a qualidade das evidências baseadas na categorização da Agency for Healthcare Research and Quality (AHRQ) dos Estados Unidos da América é classificada em seis níveis, a 
saber: nível 1, metanálise de múltiplos estudos controlados; nível 2, estudo individual com delineamento experimental; nível 3, estudo com delineamento quase-experimental como estudo sem randomização com grupo único pré e pósteste, séries temporais ou caso-controle; nível 4, estudo com delineamento não-experimental como pesquisa descritiva correlacional e qualitativa ou estudos de caso; nível 5, relatório de casos ou dado obtido de forma sistemática, de qualidade verificável ou dados de avaliação de programas; nível 6, opinião de autoridades respeitáveis baseada na competência clínica ou opinião de comitês de especialistas, incluindo interpretações de informações não baseadas em pesquisas $^{12}$.

\section{RESULTADOS}

Dos dez estudos incluídos (E1 a E10), todos originariamente na língua inglesa, nove foram conduzidos nos Estados Unidos e somente um no Reino Unido, publicados um em 2003 e os demais entre 2010 a 2020, como apresentado na Figura 3.

A descrição a seguir destaca uma breve apresentação dos aspectos metodológicos de cada estudo. No E1 foi realizado um ensaio clínico randomizado com duas intervenções em um público de 301 adolescentes entre 13 a 17 anos. A primeira intervenção, realizada através do trabalho em grupo, utilizando a entrevista motivacional para envolver o participante, incluiu resolução de problemas com o objetivo de melhorar o autocuidado, como monitoramento glicêmico e dosagem de insulina em bolus. A outra intervenção envolveu lembretes de texto no celular pessoal do adolescente para verificar a adesão.

O ensaio clínico randomizado realizado com 258 adolescentes entre 13 a 16 anos (E2) testou uma intervenção comportamental adaptativa que integrou entrevistas motivacionais e treinamento de habilidades para resolver problemas e aumentar a adesão ao autogerenciamento do diabetes.

No estudo não-experimental (E3) realizado com 55 adolescentes na faixa etária entre 13 a 18 anos e 11 profissionais da saúde, os adolescentes foram submetidos à entrevista motivacional e avaliação de comportamentos nos cuidados e autoeficácia. Os profissionais de saúde que não utilizaram linguagem aderente à entrevista motivacional, ou seja, que usaram enfrentamento e persuasão, obtiveram piores resultados quanto ao controle glicêmico e menor autoeficácia no controle do diabetes em seus pacientes.

Outro ensaio clínico randomizado (E4), realizado com adolescentes DM1 entre 13-16 anos, comparou o antes e depois da intervenção utilizando a entrevista motivacional e resolução de problemas; além do uso, por todos os participantes, de um livro educacional para pacientes com DM1, o Pink Panther Understanding Diabetes. Neste estudo, foram avaliados os principais tópicos para a gestão do cuidado como a verificação da glicose, ingestão de insulina, alimentação saudável e atividade física.

O ensaio clínico randomizado (E5) incluiu a participação de 64 adolescentes com DM1 e seus pais e/ou responsáveis, os quais foram divididos em três grupos: no primeiro grupo, tanto os pais como os adolescentes receberam a intervenção motivacional; no segundo, somente os pais receberam a intervenção; e no terceiro grupo, os adolescentes e os pais não receberam a intervenção. A intervenção visava transmitir aos pais reflexões acerca do monitoramento parental e as vantagens no tratamento desse adolescente.

No estudo piloto de uma intervenção realizada através da web com 15 adolescentes na faixa etária de 13 a 17 anos e seus familiares de área rural com diabetes tipo 1 mal controlada (E6), os participantes eram submetidos à intervenções que incluíam entrevista motivacional e princípios comportamentais cognitivos.

O ensaio clínico randomizado (E7) aplicou uma intervenção motivacional por computador em 23 adolescentes de 10 a 13 anos. Já o estudo piloto (E8) desenvolveu durante 14 semanas uma intervenção motivacional com 17 adolescentes entre 12 e 17 anos e seus pais.

O ensaio clínico controlado (E9) comparou a educação por entrevistas motivacionais e a educação estruturada em diabetes durante nove meses com 44 adolescentes com DM1. Por fim, o estudo piloto (E10) avaliou durante seis meses o efeito das sessões de entrevista motivacional na hemoglobina glicada e aspectos psicológicos de 22 adolescentes com idade entre 14 e 18 anos.

Todos os artigos destacam a entrevista motivacional como estratégia favorável no autogerenciamento dos cuidados de adolescentes com DM1, em sua maioria, relacionando a outras abordagens, conforme os tipos de estudo sinalizam os benefícios na melhoria da saúde, essencialmente, no controle da glicemia. 


\begin{tabular}{|l|l|}
\hline $\begin{array}{l}\text { Pais de origem / } \\
\text { Nível de evidência }\end{array}$ & \multicolumn{1}{|c|}{ Objetivos } \\
\hline $\begin{array}{l}\text { E1. Estados Unidos } \\
\text { da América }{ }^{13} \\
\text { Nível } 2\end{array}$ & $\begin{array}{l}\text { Avaliar o impacto da hemoglobina glicada em } \\
\text { adolescentes com DM1 por meio de duas } \\
\text { intervenções: solução de problemas e mensagens de } \\
\text { texto }\end{array}$ \\
\hline $\begin{array}{l}\text { E2. Estados Unidos } \\
\text { da América14 } \\
\text { Nível } 2\end{array}$ & $\begin{array}{l}\text { Aplicar um novo método estatístico para análise que } \\
\text { deriva uma regra de tratamento individualizado para } \\
\text { identificar participantes do Flexible Life styles } \\
\text { Empowering Change (FLEX) que podem se beneficiar } \\
\text { da intervenção com base em alterações na } \\
\text { porcentagem da hemoglobina glicada (resultado } \\
\text { primário), qualidade de vida e escore z do índice de } \\
\text { massa corporal (desfechos secundários) durante 18 } \\
\text { meses. }\end{array}$ \\
\hline
\end{tabular}

E3. Estados Unidos Explorar o uso da Entrevista Motivacional na da América ${ }^{4}$ comunicação de adolescentes e profissionais da saúde Nível $4 \quad$ com a adesão ao tratamento de DM1, autoeficácia e controle glicêmico

E4. Estados Unidos Testar a eficácia da intervenção comportamental da América ${ }^{15}$ adaptativa do Flexible Lifestyles Empowering Change Nível 2 (FLEX) nos resultados primários (hemoglobina glicada) e nos resultados secundários (psicossocial e metabólico) ao longo de 18 meses.

E5. Estados Unidos Testar a viabilidade e eficácia de uma intervenção da América ${ }^{16}$ motivacional de três sessões, no período de 6 meses, Nível 2 realizadas por computador, para promover maior monitoramento dos pais cuidadores primários de jovens adolescentes afro-americanos com DM1.

E6) Estados Unidos Examinar a viabilidade e eficácia de uma intervenção da América ${ }^{17}$ realizada através da web visando a autorregulação e Nível $4 \quad$ automonitoramento dos níveis de glicose no sangue e controle glicêmico em adolescentes com diabetes tipo 1 que vivem em áreas rurais dos EUA.

E7. Estados Unidos da América $^{18}$ Nível 4

Verificar a viabilidade de uma intervenção em computador de três sessões no intervalo de 3 a 4 meses, para melhorar o gerenciamento de diabetes entre jovens afro-americanos com diabetes tipo 1.

\section{Principais resultados}

As duas intervenções abordaram o adolescente acerca da resolução dos problemas, e, apesar de não obter mudanças nos parâmetros da hemoglobina glicada, houve um melhor controle glicêmico. Mostrou o amplo uso de celulares pelo público alvo e a intervenção de mensagem de texto foi encorajadora, diante dos lembretes dos cuidados.

Os adolescentes que integraram o grupo da intervenção com a estratégia de aconselhamento comportamental (FLEX), juntamente a entrevista motivacional e o treinamento de habilidades para resolução dos problemas, melhoraram suas habilidades e adesão na gestão do tratamento.

Os profissionais da saúde que tiveram comportamentos de não adesão, ou seja, que utilizavam enfrentamento e persuasão, obtiveram em seus pacientes o pior controle glicêmico e menor autoeficácia no controle da diabetes.

A intervenção FLEX não alterou significativamente os resultados da hemoglobina glicada, mas impactou positivamente nos fatores psicossociais.

Os adolescentes e seus pais apresentaram aumento dos conhecimentos após a entrevista motivacional e, além disso, o estudo traz que a intervenção ajudou os pais no cuidado com seus filhos

Após a intervenção educativa e a entrevista motivacional mostraram melhora do desempenho no tratamento e assim, contribuindo para o desempenho do automonitoramento glicêmico e diminuição da hemoglobina glicada.

Intervenção computadorizada com informação motivação-comportamento e a entrevista motivacional permitiram melhora no tratamento de adolescentes com DM1.

Estudo piloto mostrou melhora na frequência do

monitoramento glicêmico dos adolescentes com DM1, apontando eficácia da intervenção, o qual é o mecanismo para mudança de comportamento e motivação.

A entrevista motivacional associada a uma intervenção educativa estruturada afetou positivamente os adolescentes, melhorando os parâmetros glicêmicos, além de contribuir na motivação ao tratamento.

Após a aplicação da entrevista motivacional obteve-se melhorias nas médias da hemoglobina glicada, além de contribuição para reduzir os sentimentos negativos gerados pelo adoecimento.

E9. Estados Unidos Comparar a educação por entrevistas motivacionais e da América ${ }^{20}$ a educação estruturada em diabetes para melhorar as Nível 2 medidas psicossociais e hemoglobina glicada em adolescentes com diabetes tipo 1

E10. Reino Unido ${ }^{21}$ Obter dados preliminares sobre o impacto da Nível $4 \quad$ entrevista motivacional, uma abordagem de aconselhamento para mudança de comportamento, controle glicêmico, bem-estar e autocuidado de adolescentes com diabetes.

FIGURA 3: Caracterização dos estudos quanto à identificação e país de origem, nível de evidência, objetivos e principais resultados. Fortaleza, CE, Brasil, 2020. Fonte: Elaborado pelas autoras. 


\section{DISCUSSÃO}

Os estudos identificados mostram os benefícios da EM como intervenções profissionais junto aos adolescentes com DMI. Contudo, foi ressaltado os aspectos intervenientes desde o modo de aplicação, duração e na abordagem a este público deve ser adaptada à sua compreensão e motivação.

Nesse sentido, os estudos (E6, E8, E10) confirmaram resultados positivos no controle glicêmico e na diminuição da hemoglobina glicada durante e após a intervenção.

Resultado semelhante no estudo (E8) que mostrou efeito positivo da intervenção, com respostas no cuidado e os adolescentes aumentaram significativamente o monitoramento glicêmico com respostas de melhoria na hemoglobina glicada após a intervenção. Resultado semelhante ocorreu em outro estudo (E6), em que o automonitoramento da glicemia aumentou expressivamente no final do tratamento ativo e durante sua manutenção, o nível da hemoglobina glicada diminuiu.

No estudo piloto realizado no Reino Unido (E10), observou-se o efeito da intervenção sobre a hemoglobina glicada média, que diminuiu de 10,8\% para 9,7\% durante o estudo e permaneceu menor após o período de intervenção. Contudo, observou-se que não houve redução nos valores de hemoglobina glicada no grupo que não recebeu a intervenção da entrevista motivacional.

Salienta-se achados diferentes no estudo (E1), pois não foi encontrada diferença significativa na hemoglobina glicada ao longo do tempo, por grupo de estudo, considerando desfecho primário de 12 meses. Assim como, no ensaio clínico randomizado (E4), a hemoglobina glicada não apresentou diferença estatisticamente significante entre o grupo de intervenção e grupo controle.

Também se observou em outro ensaio clínico randomizado (E1) que o grupo de intervenção que utilizava a entrevista motivacional não obteve melhora do controle glicêmico. Porventura, nesse mesmo estudo, afirmou-se que, apesar de não haver diferença de $\mathrm{HbA1c}$ por grupo de estudo, houve uma maior resposta aos lembretes de mensagens de texto para verificar a glicemia, resultando em melhor controle glicêmico e nenhuma deterioração do HbA1c.

Obtiveram-se respostas positivas no estudo (E9) ao comparar a entrevista motivacional e a educação estruturada em diabetes para melhorar as medidas psicossociais e a hemoglobina glicada em adolescentes com diabetes tipo 1. Em seis meses de acompanhamento, o grupo de educação estruturada obteve melhor resposta que o grupo submetido à EM. Afirmou-se nos procedimentos metodológicos que os três profissionais que aplicaram a entrevista motivacional foram treinados somente durante dois dias em oficinas.

No grupo de educação estruturada foi utilizada uma lista abrangente de recomendações da American Diabetes Association (ADA), conteúdo básico adotada em suas rotinas. Tais resultados supõem que os procedimentos da entrevista motivacional não tenham sido bem assimilados pelos profissionais, pois tiveram um contato breve, indicando possível inconsistência na aplicação da intervenção. Diante disso, supõe-se a necessidade de capacitação estendida aos profissionais para aplicação da EM.

Ao passo que no estudo (E10) os pesquisadores que aplicaram a entrevista motivacional, participaram de uma capacitação por três meses de oficinas e treinamentos com vídeo, além da supervisão semanal durante o período de aplicação da intervenção. O resultado da pesquisa utilizando a EM apresentou resposta positiva na diminuição da hemoglobina glicada durante e após aplicação da intervenção.

Uma meta-análise ${ }^{22}$, sugeriu que a entrevista motivacional produz um efeito pequeno, mas significativo em vários comportamentos de saúde entre os jovens, indicando que, no geral, a EM alcança os objetivos. Além disso, os autores analisaram que os efeitos da EM parecem ser duráveis, pois não houve evidência de uma diminuição estatisticamente significativa no tamanho do efeito ao longo do tempo de acompanhamento.

Este estudo 22 ressalta que quando os profissionais de saúde não têm experiências na aplicação da EM, não alcançam resultados positivos sobre a adesão e autoeficácia no tratamento de diabetes de seus pacientes e, consequentemente, no controle da hemoglobina glicada. Portanto, para garantir a qualidade da aplicação da EM faz-se necessário treinamento e presença de supervisão contínua, possibilitando maior empoderamento com o método de intervenção e maior efeito nos resultados.

Complementa-se que estudos (E4 e E10) mostraram desfechos psicossociais positivos no uso da EM. O estudo (E10) realizado com 22 adolescentes observou uma redução no medo da hipoglicemia e melhor convivência com a doença. Outro, (E4), apesar da hemoglobina glicada não ter apresentado diferença estatisticamente significante entre o grupo de intervenção e grupo controle, a intervenção foi associada a melhores escores de motivação e qualidade de vida geral, resolução de problemas, autogerenciamento do diabetes, redução das barreiras de adesão ao autogerenciamento e melhoria nas relações familiares. 
Observou-se que a EM era realizada, principalmente, por encontros presenciais, sendo adotados também os meios digitais. No estudo (E10) intercalaram encontros presenciais e ligação telefônica. Três estudos (E5, E6 e E7) utilizaram computadores e web para a intervenção. As intervenções foram realizadas no período de um a 18 meses, sendo quatro desses no período de seis meses (E5, E6, E9 e E10); outros quatro estudos, no período de nove a 18 meses (E1, E2, E4 e E7); e dois estudos (E3 e E8) no período entre um e três meses. O número de sessões variou de três a 25 sessões, quando descritas no estudo, pois alguns estudos (E2 e E3) não especificaram o número total de sessões. A média foi de três a cinco sessões (E1, E4, E5 e E7).

Quanto à influência da EM no autogerenciamento do cuidado ao adolescente com DM1, em diversos estudos foram observados resultados positivos (E2, E4, E6, E7 e E10) como redução do medo de hipoglicemia, melhor convivência com a doença, melhores escores de motivação e resolução de problemas e ainda na melhora do IMC. Outro efeito significativo foi encontrado na pesquisa (E6) que obteve melhoria no desempenho das tarefas de memória e controle inibitório (habilidade para inibir ou controlar respostas impulsivas ou automáticas). Assim, os pais relataram, menos problemas com a gestão dos processos cognitivos.

A pesquisa (E7) que aplicou a EM baseada no modelo de informação, motivação e habilidades de mudança de comportamento em saúde dos jovens com DM1 verificou a viabilidade da intervenção para melhorar o gerenciamento dos cuidados. Os participantes consideraram a estratégia útil para motivar o controle do diabetes e empoderá-los com o apoio da família.

Confirma-se na meta-análise 22 a eficácia da EM na população infantojuvenil, indicando que é uma intervenção eficaz e apropriada para direcionar as mudanças de comportamento de saúde destes sujeitos, melhorando o autogerenciamento dos cuidados com a diabetes. É compreensível que quando os profissionais apoiam a autonomia do paciente de forma colaborativa, permite que o paciente seja o condutor da mudança e torne-se propenso a mudar, assumindo seus cuidados ${ }^{23}$.

Desse modo, reforça o estudo (E7) que intervenções envolvendo motivação têm potencial de melhorar a saúde do adolescente. Outrossim, uma pesquisa (E5) que incluiu intervenções com os pais e os adolescentes observou que os adolescentes não obtiveram resultados tão significativos, obtendo-se melhores resultados em seus pais. Contudo, esse mesmo estudo ressalta que supervisão dos pais aumentava à medida que diminuía o conflito familiar e pais encontraram resistência significativa do adolescente, possivelmente, por terem parado de monitorar. É compreensível que o conflito familiar pode desenvolver efeitos adversos, como a diminuição da autoestima do adolescente.

Na avaliação dos estudos primários, foram observados apenas cinco estudos (E1, E2, E4, E5 e E9) com evidência forte, o que indica a necessidade de conduzir pesquisas com maior nível de evidências para que se possa afirmar a eficácia da entrevista motivacional no autogerenciamento do cuidado de adolescente com diabetes mellitus tipo 1.

Apontam-se algumas limitações nesta revisão, tais como a metade dos estudos configurava-se como pesquisa descritiva, que por si traz fatores não controlados que poderiam implicar nos resultados da Entrevista Motivacional na comparação a outras estratégias educativas. Além disso, analisar dados de estudos primários conduzidos por diferentes abordagens e procedimentos variados nas intervenções é desafiador, com um possível viés na construção dos resultados.

\section{CONCLUSÃO}

Os estudos identificados e analisados sobre a EM e sua influência no autogerenciamento do cuidado de adolescentes com DM1 trazem variações de respostas comportamentais com impactos sobre o controle nos níveis glicêmico e melhora do IMC. Entretanto, por serem intervenções comportamentais e cognitivas, muitos aspectos intervenientes e subjetivos podem influenciar nos resultados.

Ainda assim, a pesquisa sugere que o uso de entrevista motivacional tem aumentado como intervenção promissora e de efeito duradouro para promover o autogerenciamento do adolescente com diabetes, podendo contribuir para a autoeficácia. Por conseguinte, para maior efeito da intervenção, diversos aspectos devem ser considerados, dentre eles, a capacitação do profissional que aplica a técnica da entrevista motivacional e o tempo de aplicação.

Conclui-se que alguns estudos não conseguiram mostrar significância estatística da EM sobre a melhora da hemoglobina glicada, mas mostraram algum impacto, seja fisiológico ou psicossocial como o autogerenciamento do cuidado na finalidade educativa. Contudo, os profissionais devem ser treinados a utilizar a EM, para obter respostas terapêuticas melhores, trazendo, portanto, benefícios diretos no tratamento e na gestão dos cuidados, favorecendo a autonomia do adolescente com diabetes. 


\section{REFERÊNCIAS}

1. International Diabetes Federation. Diabetes Atlas. 9. ed. Brussels: Belgium. 2019. [cited 2020 Jul 12]. Available from: https://www.idf.org/e-library/epidemiology-research/diabetes-atlas/159-idf-diabetes-atlas-ninth-edition-2019.html.

2. Ellis D, Carcone A, Ondersma S, Naar-King S, Dekelbab B, Moltz K. Brief computer-delivered intervention to increase parental monitoring in families of african american adolescents with type 1 diabetes: A randomized controlled trial. Telemedi J E Health [Internet]. 2017 [cited 2020 Jul 23]; 23(6):493-502. DOI: https://doi:10.1089/tmj.2016.0182.

3. Luke S, Richards L. Motivational Interviewing: A tool to open dialogue with teens with type 1 diabetes mellitus. J Pediatr Nurs [Internet]. 2018 [cited 2020 Jul 02]; 40:89-91. DOI: https://doi.org/10.1016/j.pedn.2018.02.014.

4. Sociedade Brasileira de Diabetes. Diretrizes da sociedade brasileira de diabetes 2019-2020. [Internet] Editora científica. $491 \mathrm{p}$. 2019 [cited 2020 Sep 10]. Available from: https://portaldeboaspraticas.iff.fiocruz.br/biblioteca/diretrizes-da-sociedadebrasileira-de-diabetes-2019-2020/.

5. Thompson D, Chair S, Chan S, Astin F, Davidson P, Ski C. Motivational interviewing: A useful approach to improving cardiovascular health? J Clin Nurs [Internet]. 2011 [cited 2020 Jul 15]; 20(9-10):1236-44. DOI: https://doi.org/10.1111/j.13652702.2010.03558.x.

6. Williams AF, Manias E, Cross WM, Crawford KE. Motivational interviewing to explore culturally and linguistically diverse people's comorbidity medication self-efficacy. J Clin Nurs [Internet]. 2015 [cited 2020 Jul 11]; 24(9-10):1269-79. DOI: https://doi.org/10.1111/jocn.12700.

7. Caccavale LJ, Corona R, LaRose JG, Mazzeo SE, Sova AR, Bean MK. Exploring the role of motivational interviewing in adolescent patient-provider communication about type1 diabetes. Pediatr Diabetes. [Internet]. 2019 [cited 2020 Aug 23]; 20(2):217-25. DOI: https://doi.org/10.1111/pedi.12810.

8. Duran L. Motivating Health: Strategies for the Nurse Practitioner. J Am Acad Nurse Pract. [Internet]. 2003 [cited 2020 Aug 12]; 15(5):200-5. DOI: https://doi.org/10.1111/j.1745-7599.2003.tb00359.x.

9. Mendes KD, Silveira RC, Galvão CM. Revisão integrativa: Método de pesquisa para a incorporação de evidências na saúde e na enfermagem. Texto Context Enferm [Internet] 2008 [cited 2020 Jul 12] 17(4):758-64. Available from: https://www.redalyc.org/comocitar.oa?id=71411240017.

10. Soares CB, Hoga LAK, Peduzzi M, Sangaleti C, Yonekura T, Silva DRAD. Integrative review: Concepts and methods used in nursing. Rev. Esc. Enferm. USP [Internet]. 2014 [cited 2020 Jul 17]; 48(2):335-45. DOI: https://doi.org/10.1590/s00806234201400002000020 .

11. Santos CMC, Pimenta CAM, Nobre MRC. The PICO strategy for the research question construction and evidence search. Rev. LatinoAm. Enfermagem [Internet]. 2007 [cited 2020 Jul 17]; 15(3):508-11. DOI: https://doi.org/10.1590/S0104-11692007000300023.

12. Moher D, Liberati A, Tetzlaff J, Altman DG, Group P. Preferred reporting items for systematic reviews and meta-analyses: The PRISMA statement. PLoS Med [Internet]. 2009 [cited 2020 Jul 17]; 6(7): e1000097. DOI: https://doi.org/10.1371/journal.pmed.1000097.

13. Galvão CM. Níveis de evidência. Acta paul. Enferm [Internet]. 2006 [cited 2020 Jul 02]; 19(2):v-vii. DOI: https://doi.org/10.1590/S0103-21002006000200001.

14. McGill D, Laffel L, Volkening L, Butler D, Levy W, Wasserman R, Anderson B. (2020). Text message intervention for teens with type 1 diabetes preserves hba1c: Results of a randomized controlled trial. Diabetes Technol Ther [Internet]. 2020 [cited 2020 Jul 14]; 22(5):374-82. DOI: https://doi.org/10.1089/dia.2019.0350.

15. Kahkoska A, Lawson M, Crandell J, Driscoll K, Kichler J, Seid M, Maahs D, Kosorok M, Mayer-Davis E. Assessment of a precision medicine analysis of a behavioral counseling strategy to improve adherence to diabetes self-management among youth A post hoc analysis of the FLEX Trial. JAMA Netw Open [Internet]; 2019 [cited 252020 Jul]; 2(5):e195137. DOI: https://doi.org/10.1001/jamanetworkopen.2019.5137.

16. Mayer-Davis E, Maahs D, Seid M, Crandell J, Bishop F, Driscoll K, Hunter C, Kichler J, Stantiford D, Thomas J. The flexible lifestyles empowering change (FLEX) intervention for adolescents with type 1 diabetes: Randomized clinical trial results for effect on metabolic status, diabetes related behaviors, and quality of life. Lancet Child Adolesc Health [Internet] 2018 [cited 2020 Jul 16] 2(9), 635-46. DOI: https://doi.org/10.1016/S2352-4642(18)30208-6.

17. Lansing A, Stanger C, Budney A, Christiano A, Casella S. Pilot study of a web-delivered multicomponent intervention for rural teens with poorly controlled type 1 diabetes. Journal of Diabetes Research [Internet]. 2016 [cited 2020 Jul 10]; 7485613. DOI: http://dx.doi.org/10.1155/2016/7485613.

18. Rajkumar D, Ellis D, May D, Carcone A, Naar- King S, Ondersma S, Moltz K. Computerized intervention to increase motivation for diabetes self-management in adolescents with type 1 diabetes. Health Psychol. Behav. Med. [Internet]. 2015 [cited 2020 Jul 10]; 3(1):236-50. DOI: https://doi:10.1080/21642850.2015.1079716.

19. Stanger C, Ryan S, Delhey L, Thrailkill K, Li Zhonge, Li Zhigang, Budney A. A multicomponent motivational intervention to improve adherence among adolescents with poorly controlled type 1 diabetes: A pilot study. J. Pediatr. Psychol. [Internet]. 2013 [cited 2020 Jul 12]; 38(6):629-37. DOI: https://doi.org/10.1093/jpepsy/jst032.

20. Wang Y, Stewart S, Mackenzie M, Nakonezny P, Edwards D, White P. A randomized controlled trial comparing motivational interviewing in education to structured diabetes education in teens with type 1 diabetes. Diabetes Care [Internet]. 2010 [cited 2020 Jul 14]; 33(8):1741-3. DOI: https://doi.org/10.2337/dc10-0019.

21. Channon S, Smith V, Gregory J. A pilot study of motivational interviewing in adolescents with diabetes. Arch Dis Child. [Internet]. 2003 [cited 2020 Jul 29]; 88:680-3. DOI: https://doi.org/10.1136/adc.88.8.680. 
22. Gayes LA, Steele RG. A meta-analysis of motivational interviewing interventions for pediatric health behavior change. J. Consult. Clin. Psychol. [Internet]. 2014 [cited 2020 Jul 24]; 82(3):521-35. DOI: https://doi.org/10.1037/a0035917.

23. Pollak KI, Alexander SC, Coffman CJ, Tulsky J A, Lyna P, Dolor RJ, James IE, Brouwer RJN, Manusov JRE, Østbye T. Physician communication techniques and weight loss in adults project CHAT. Am. J. Prev. Med. [Internet]. 2010 [cited 2020 Jul 24]; 39(4):321-8. DOI: https://doi.org/10.1016/j.amepre.2010.06.005. 